Provided for non-commercial research and education use. Not for reproduction, distribution or commercial use.

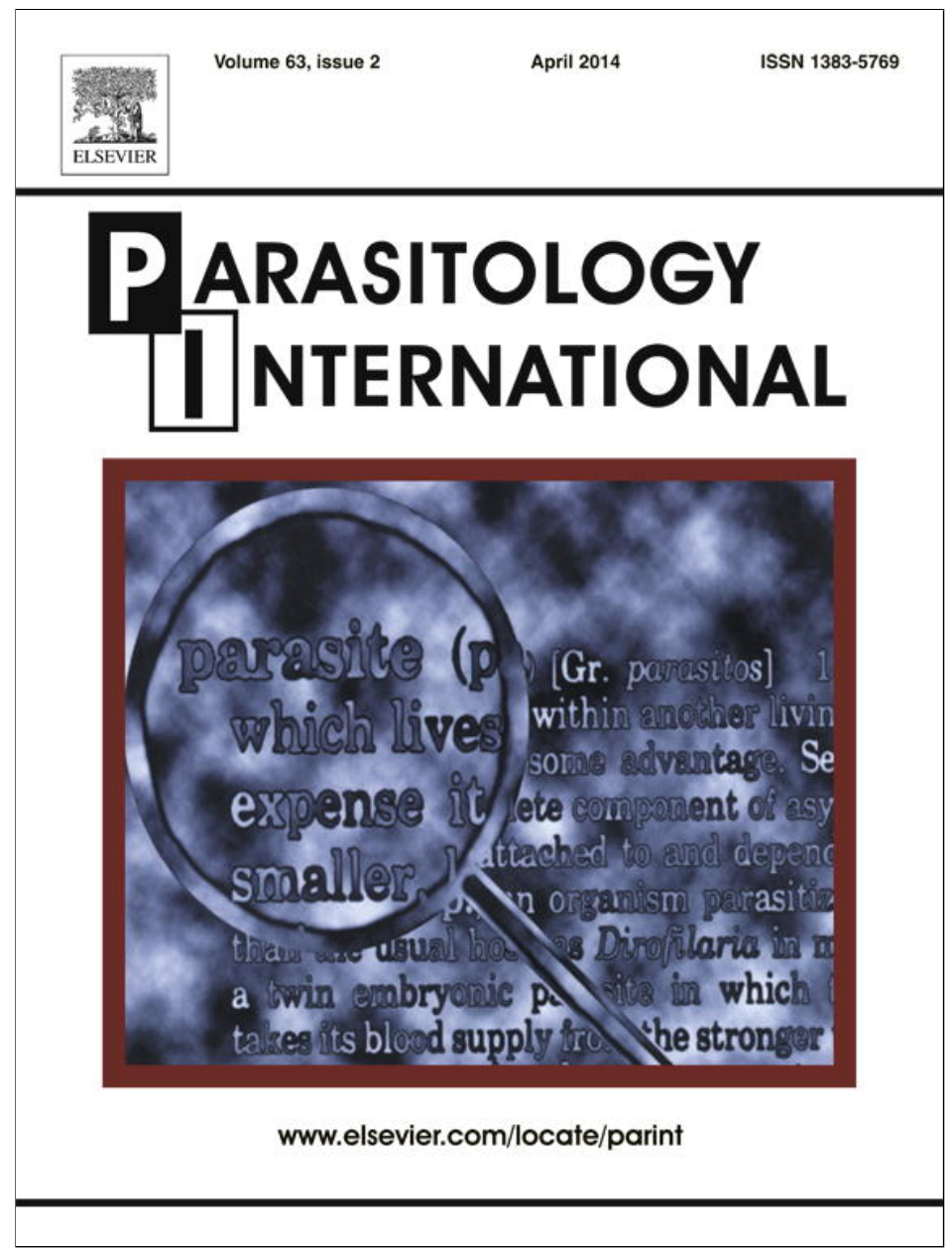

This article appeared in a journal published by Elsevier. The attached copy is furnished to the author for internal non-commercial research and education use, including for instruction at the authors institution and sharing with colleagues.

Other uses, including reproduction and distribution, or selling or licensing copies, or posting to personal, institutional or third party websites are prohibited.

In most cases authors are permitted to post their version of the article (e.g. in Word or Tex form) to their personal website or institutional repository. Authors requiring further information regarding Elsevier's archiving and manuscript policies are encouraged to visit: 


\title{
Genetic characterization of Giardia duodenalis by sequence analysis in humans and animals in Pemba Island, Tanzania
}

\author{
V. Di Cristanziano ${ }^{\text {a,b,*, }}$, M. Santoro ${ }^{\text {b,c }}$, F. Parisi $^{\text {d }}$, M. Albonico ${ }^{\text {d }}$, M.A. Shaali ${ }^{\text {e, D. Di Cave }}{ }^{\text {b }}$, F. Berrilli ${ }^{\text {b }}$ \\ a Institute of Virology, University of Cologne, Fürst-Pückler-Str. 56, 50935 Cologne, Germany \\ b Department of Experimental Medicine and Surgery, University of Rome "Tor Vergata", Via Montpellier 1, 00133 Rome, Italy \\ c Parasitology Unit, Bambino Gesù Children's Hospital, IRCCS, Piazza Sant'Onofrio 4, 00165 Rome, Italy \\ d Ivo de Carneri Foundation, Viale Monza 44, 20127 Milan, Italy \\ e Public Health Laboratory (Pemba)-Ivo de Carneri, P.O. Box, TZ-122 Wawi, Chake Chake, Tanzania
}

\section{A R T I C L E I N F O}

\section{Article history:}

Received 30 April 2013

Received in revised form 4 November 2013

Accepted 11 November 2013

Available online 19 November 2013

\section{Keywords:}

Giardia duodenalis

Genetic characterization

ssu-rDNA

gdh

Tanzania

\begin{abstract}
A B S T R A C T
Giardia duodenalis represents one of the most widespread human enteric parasites: about 200 million people in Asia, Africa and Latin America are infected. Giardia exerts a deep impact on public health because of high prevalence and possible effects on growth and cognitive functions in infected children. The major aim of this study was to detect and genetically characterize G. duodenalis in both human and animal fecal samples collected in Pemba Island, in the archipelago of Zanzibar (Tanzania), in order to deepen the knowledge of genotypes of Giardia in this area.

Between October 2009 and October 2010, we collected 45 human fecal samples from children from 2 primary schools and 60 animal fecal samples: 19 from zebus (Bos primigenius indicus) and 41 from goats (Capra hircus). Detection and genetic identification were performed by multilocus analysis of ssu-rDNA and gdh genes. In humans we found a higher prevalence of assemblage B (sub-assemblage BIV), in goats of assemblage E and in zebus of assemblage A. Our study represents an important contribution to the epidemiological knowledge of $G$. duodenalis in this area of Tanzania.
\end{abstract}

(C) 2013 Elsevier Ireland Ltd. All rights reserved.
Giardia duodenalis (syn. G. intestinalis; G. lamblia) is a flagellated protozoan that infects the intestine of a wide range of vertebrate hosts and the only species of Giardia found in humans. Genetic studies have demonstrated that $G$. duodenalis is a multi-species complex comprising at least seven assemblages, identified from $A$ to $G$ [1]. In addition to these described assemblages, several novel genotypes have been reported including the assemblage $\mathrm{H}$ proposed by Lasek-Nesselquist et al. in 2010 [2]. Only assemblages $\mathrm{A}$ and $\mathrm{B}$ are capable of infecting humans but they are also found in a wide range of other mammalian hosts, so that they are considered potentially zoonotic. The distribution of the assemblages A and B is different in several studies and countries. In addition, a significant intraassemblage genetic variability is recognized in both assemblages [1].

G. duodenalis is a common cause of diarrheal disease in humans, particularly among disadvantaged groups where recurrent infections contribute to growth deficits and malnutrition, especially in children in developing countries [3]. The human prevalence rates range from 2 $7 \%$ in developed countries to $20-30 \%$ in most developing countries [4], due to poor hygiene and limited access to safe water supply. In September 2004, Giardia was included in the Neglected Diseases Initiative of the WHO [5]. Little data on the prevalence of G. duodenalis are available

* Corresponding author at: Institute of Virology, University of Cologne, Fürst-PücklerStr. 56, 50935, Cologne, Germany. Tel.: + 49 2214783927; fax: + 492214783902.

E-mail address: veronica.di-cristanziano@uk-koeln.de (V. Di Cristanziano). from sub-Saharan Africa and a genetic characterization of the parasite in these regions has been rarely performed. On the other hand collection of molecular data from endemic areas is necessary to better understand host and environmental interactions within the disease [6].

Considering the absence of molecular data related to G. duodenalis in Tanzania, in the present study we have genetically characterized at two loci isolates of $G$. duodenalis collected from humans and animals on Pemba Island, in order to identify the circulating assemblages and sub-assemblages and the transmission dynamics in this area.

Pemba Island is the second largest island of the archipelago of Zanzibar, located in the Indian Ocean south of the equator and $48 \mathrm{~km}$ from the eastern coast of the African continent. Between October 2009 and October 201045 fecal samples were obtained from humans in pediatric age (7-12 years) attending two primary schools in the district of Chake Chake, the main city of Pemba Island. In the same period, 60 samples from animals (41 from goat - Capra hircus - and 19 from zebu cattle - Bos primigenius indicus) were collected in a day, directly from the soil, immediately after observed defecation from each host in Kojani, a small village on the coast and separated from the island of Pemba by a narrow stretch of sea tidal. Children were selected at random from schools, located nearby the Public Health Laboratory Ivo de Carneri (PHL-IdC) where the first part of the study was carried out. Animals were selected from Kojani Island by local veterinary personnel. A continuous movement of people and livestock inter- and intra- the two 
islands was present. All samples were smeared on filter paper $\left(8 \mathrm{~cm}^{2}\right)$, dried at room temperature for about $30 \mathrm{~min}$ and stored in plastic containers at room temperature before being transported to Italy as previously described in Berrilli et al. [7].

Informed verbal assent was obtained from children's parents or legal guardians. All investigations were performed in concordance with the WMA Helsinki Declaration (Edinburgh 2000). Ethical clearance was obtained by the Zanzibar Health Research Council.

The samples were carried to the University of Rome Tor Vergata where the molecular analysis was performed. DNA extraction was performed by cutting about $2 \mathrm{~cm}^{2}$ of the smeared filter paper and using it for the extraction by QIAamp DNA Micro Kit (QIAgen, Gmbh, Hilden, Germany) in according to the manufacturer's recommendations. The final eluate was stored at $-20^{\circ} \mathrm{C}$. In order to unequivocally assigned Giardia isolates at the assemblage level and identify sub-assemblages, PCR was carried out to amplify a 130 bp region from the small subunit ribosomal RNA (ssu-rDNA) [8], and a 432 bp region of the glutamate dehydrogenase ( $g d h)$ gene [9]. Positive (Giardia DNA) and negative (no template added) control samples were used in all PCR runs. PCR products were separated by electrophoresis in 1\% agarose gel and amplicons were purified using the NucleoSpin ${ }^{\circledR}$ Extract kit (MachereyNagel GmbH \& Co. KG, Germany). Both strands were sequenced by the Bio-Fab Research s.r.l. (Rome, Italy). Sequences were edited using the FinchTV 1.4 software (Geospiza, Inc., Seattle, WA). Consensus sequence was determined by alignment of respective forward and reverse sequences. Assignment to assemblage and sub-assemblage of G. duodenalis isolates was carried out by sequence comparison (ssu-rDNA) and phenetic analysis $(g d h)$. Multiple alignments were performed using ClustalW2 software for DNA against known sequences available in GenBank for Giardia assemblages. Phenetic analysis was performed using the software MEGA5, conducted using the Tamura 3-parameter method and the phenetic tree was constructed by the Neighbor-Joining algorithm. Bootstrap values were calculated by analyzing 1000 replicates. All gdh sequences obtained in this study were deposited in the GenBank database and are available under the accession numbers: KF468653-KF468671.

As for humans, ssu-rDNA amplicons were obtained from 25 of the 45 samples, whereas 17 resulted positive in the $g d h$ PCR. Eleven out of 25 ssu-rDNA positive samples were negative for $g d h$ gene analysis, while 3 out of 20 ssu-rDNA negative samples were positive to gdh locus (Table 1). Sequences and phenetic analyses allowed the assignment of 6 isolates to assemblage A ( 3 of them to sub-assemblage AII) and 22 isolates to assemblage B, particularly 4 isolates to sub-assemblage BIII and 7 to sub-assemblage BIV. Three isolates K043 (KF468658), K051 (KF468659) and K060 (KF468663) were identified as belonging to the assemblage B but could not unambiguously be assigned to subassemblage level. In particular, their sequences showed several diagnostic nucleotides typical of BIII and some typical of BIV; moreover, the sequence K043 included one double peak (C/T). The high substitution rate in $g d h$ sequences has been previously reported to limit the clearcut identification of Giardia isolates at sub-assemblage level within assemblage B [10].

Concerning animals, 9/41 samples from goats and 4/19 from zebus were found Giardia positive. The genetic characterization of goat isolates made it possible to assign 6 samples to the host-specific assemblage $\mathrm{E}$, two samples to assemblage B ( 1 of them to sub-assemblage BIV) and 1 sample to assemblage $A$. Three isolates from zebus were found to belong to assemblage $A$ and 1 to assemblage $B$ (sub-assemblage BIV) (Table 1). Fig. 1 shows the phenetic tree forming two well-defined clusters, one corresponding to the assemblage A (sub-assemblage AII), the other including isolates matching which sequences including different subtypes of assemblage B (BIII and BIV).

No discrepancies were observed in assemblage types as determined by sequencing of the two genes in all 41 human and animal isolates, indicating single assemblage infections.

G. duodenalis has a cosmopolitan distribution. In developing countries, giardiasis is highly endemic in humans, especially in children [3]. On Pemba Island, prevalence of infection has been assessed during 3 epidemiological surveys among the general population, in particular school-aged children. In 1984 Pampiglione et al. [11] carried out a copro-parasitological study on 413 fecal samples and G. duodenalis was detected by microscopic examination in 23 patients (5.6\%). Few years later Albonico and colleagues [12] reported a prevalence of $6.6 \%$ among children aged 9-17 years. Recently, the prevalence of Giardia in school-aged children was $16.4 \%$ [13].

In spite of its endemicity, to the best of our knowledge this is the first study on molecular characterization of G. duodenalis isolates of different origins conducted in Tanzania.

With regard to humans, by the sequencing of ssu-rDNA and gdh loci, we have assigned a higher number of isolates to the assemblage $B$, subassemblage BIV, known for its preference of human hosts [14]. As for assemblage A 3 isolates were assigned to the sub-assemblage AII, also apparently more specific for humans $[6,14]$. In no case were highlighted animal-specific assemblages (from $C$ to $G$ ). The existing data on human genotypes characterized in African countries are still limited and fragmented, covering Egypt [15,16], Ethiopia [17], Western Sahara [18], Guinea Bissau [19] and Cote d'Ivoire [7]. The distribution of assemblages observed among our samples does not differ considerably from those observed in other African countries where a higher rate for assemblage $B$ is already known, e.g. in Côte d'Ivoire [7] and Egypt [15]. Concerning Giardia infection in animals, the main presence of assemblage $\mathrm{E}$ found in goats and the identification of 3 out of 4 Giardia isolates from zebus as assemblage A is not surprising, since both host-adapted and zoonotic assemblages are reported worldwide in goats and livestock [20].

In the last decades, the studies of molecular epidemiology have revealed a wide variation in the distribution of assemblages and sub-

Table 1

Summary of genotyping results of Giardia duodenalis samples from humans and animals at the level of assemblage and sub-assemblage.

\begin{tabular}{|c|c|c|c|c|c|c|c|c|}
\hline \multicolumn{6}{|l|}{ Humans } & \multicolumn{3}{|l|}{ Animals } \\
\hline Identification code & ssu-rRNA & gdh & Identification code & ssu-rRNA & gdh & Identification code & ssu-rRNA & gdh \\
\hline A022 & B & $\mathrm{B}(\mathrm{IV})$ & K053 & B & - & G05 & E & - \\
\hline A031 & B & $\mathrm{B}(\mathrm{IV})$ & K054 & B & B (III) & G07 & E & - \\
\hline A035 & A & - & K055 & A & $\mathrm{A}(\mathrm{II})$ & G08 & B & - \\
\hline A039 & A & $\mathrm{A}(\mathrm{II})$ & K057 & B & $\mathrm{B}(\mathrm{III})$ & G11 & E & - \\
\hline A045 & B & - & K060 & B & B & G16 & A & - \\
\hline K009 & A & $\mathrm{A}(\mathrm{II})$ & K062 & B & - & G17 & - & $\mathrm{B}(\mathrm{IV})$ \\
\hline K012 & A & - & K063 & B & $\mathrm{B}(\mathrm{IV})$ & G22 & E & - \\
\hline K026 & B & - & K064 & - & $\mathrm{B}(\mathrm{IV})$ & G23 & $\mathrm{E}$ & - \\
\hline K031 & B & - & K066 & B & B (III) & G36 & $\mathrm{E}$ & - \\
\hline K035 & $\mathrm{B}$ & B (IV) & K067 & A & - & Z09 & A & - \\
\hline K043 & B & B & K070 & B & - & Z10 & A & - \\
\hline K047 & B & - & K071 & - & B (IV) & Z14 & B & B (IV) \\
\hline К049 & B & - & K072 & B & B (III) & Z19 & A & - \\
\hline K051 & B & B & K073 & - & $\mathrm{B}$ (IV) & & & \\
\hline
\end{tabular}




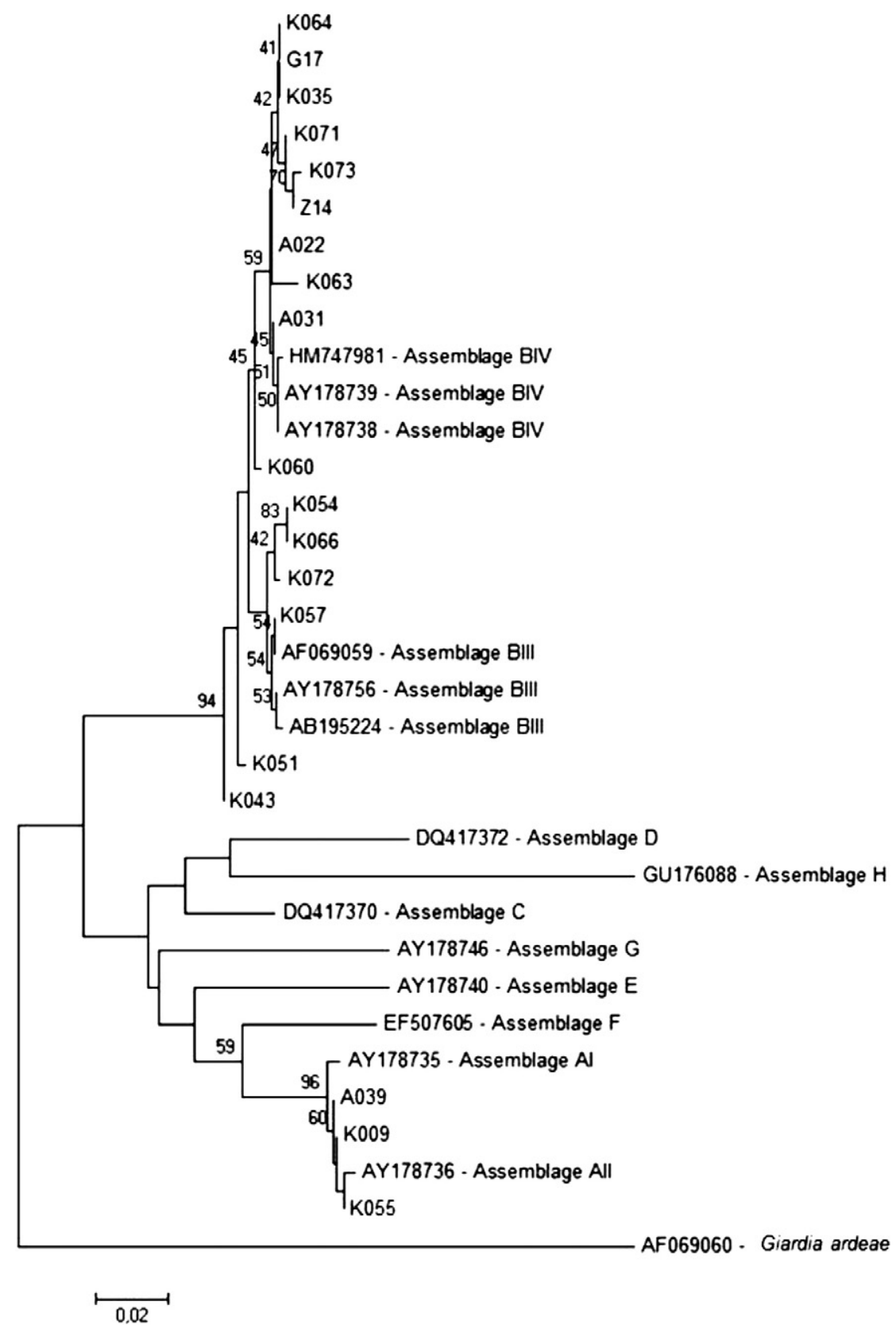

Fig. 1. Phenetic relationships inferred using the Neighbor-Joining method of the consensus gdh gene sequences obtained from PCR positive isolates.

assemblages among human and animal populations in different geographical areas worldwide, likely in correlation to the different transmission cycles identifiable at local scale [21]. Comparison of assemblages and sub-assemblages identified in humans, goats and zebus on Pemba Islands seems related to separated transmission cycles suggesting that zoonotic risk of Giardia transmission could have a minor impact on human giardiasis in the studied area. The paucity of data, however, does not yet provide enough information to better understand the epidemiological meaning of our finding that may simply reflect a sample bias.

This study has contributed to add new data about Giardia assemblages on Pemba Island and has improved the knowledge of the occurrence of these pathogens among humans and animals. Overall, the relatively high level of infection observed may pose a specific public health issue reflecting the poor availability of sanitation where people live, characterized particularly by limited access to safe water supplies and a high degree of environmental contamination due to the lack of fecal and waste disposal. More Giardia samples from much broader geographical localities of sub-Saharan Africa and from both humans and animals should be employed in further studies to better clarify the epidemiology and the zoonotic risk of giardiasis in endemic areas.

\section{Acknowledgments}

This study had not been possible without the enthusiastic participation of teachers and children from the schools of Al-Sadik and Wawi of Pemba Island. Special thanks are extended to the laboratory staff of the Public Health Laboratory Ivo de Carneri (Pemba Island, Tanzania) and of the Parasitology Unit of the General Hospital Tor Vergata (Rome, Italy).

\section{References}

[1] Plutzer J, Ongerth J, Karanis P. Giardia taxonomy, phylogeny and epidemiology: facts and open questions. Int J Environ Health 2010;213:321-33. 
[2] Lasek-Nesselquist E, Welch DM, Sogin ML. The identification of a new Giardia duodenalis assemblage in marine vertebrates and a preliminary analysis of G. duodenalis population biology in marine systems. Int J Parasitol 2010;40: 1063-74.

[3] Thompson RCA, Monis PT. Variation in Giardia: implication for taxonomy and epidemiology. Adv Parasitol 2004;58:69-137.

[4] Adam RDA. The Giardia lamblia genome. Int J Parasitol 2001;30:375-84.

[5] Savioli L, Smith H, Thompson A. Giardia and Cryptosporidium join the 'Neglected Diseases Initiative'. Trends Parasitol 2006;22:203-8.

[6] Traub RJ, Monis PT, Robertson ID. Molecular epidemiology: a multidisciplinary approach to understanding parasitic zoonoses. Int J Parasitol 2005;35: 1295-307.

[7] Berrilli F, D'Alfonso R, Giangaspero A, Marangi M, Brandonisio O, Kaboré Y, et al. Giardia duodenalis genotypes and Cryptosporidium species in humans and domestic animals in Côte d'Ivoire: occurrence and evidence for environmental contamination. Trans R Soc Trop Med Hyg 2012;106:191-5.

[8] Read C, Walters J, Robertson ID, Thompson RCA. Correlation between genotype of Giardia duodenalis and diarrhoea. Int J Parasitol 2002;32:229-31.

9] Read CM, Monis PT, Thompson RC. Discrimination of all genotypes of Giardia duodenalis at the glutamate dehydrogenase locus using PCR-RFLP. Infect Genet Evol 2004;4:125-30.

[10] Wielinga C, Ryan U, Andrew Thompson RC, Monis P. Multi-locus analysis of Giardia duodenalis intra-Assemblage B substitution patterns in cloned culture isolates suggests sub-assemblage B analyses will require multi-locus genotyping with conserved and variable genes. Int J Parasitol 2011;41:495-503.

[11] Pampiglione S, Visconti S, Stefanini A. Human intestinal parasites in sub-Saharan Africa. III. Pemba Island (Zanzibar-Tanzania). Parassitologia 1987;29:27-35.
[12] Albonico M, De Carneri I, Di Matteo L, Ghiglietti R, Toscano P, Uledi MK, et al. Intestinal parasitic infections of urban and rural children on Pemba Island: implications for control. Ann Trop Med Parasitol 1993;87:579-83.

[13] Speich B, Marti H, Ame SM, Ali SM, Bogoch II, Utzinger J, et al. Prevalence of intestinal protozoa infection among school-aged children on Pemba Island, Tanzania, and effect of single-dose albendazole, nitazoxanide and albendazole-nitazoxanide. Parasit Vectors 2013:4-3.

[14] Feng Y, Xiao L. Zoonotic potential and molecular epidemiology of Giardia species and giardiasis. Clin Microbiol Rev 2011;24:110-40.

[15] Foronda P, Bargues MD, Abreu-Acosta N, Periago MV Valero MA, Valladares B, et al. Identification of genotypes of Giardia intestinalis of human isolates in Egypt. Parasito Res 2008;103:1177-81.

[16] Helmyz MM, Abdel-Fattah HS, Rashed L. Real-time PCR/RFLP assay to detect Giardia intestinalis genotypes in human isolates with diarrhea in Egypt. J Parasitol 2009;95:1000-4.

[17] Gelanew T, Lalle M, Hailu A, Pozio E, Cacciò SM. Molecular characterization of human isolates of Giardia duodenalis from Ethiopia. Acta Trop 2007;102:92-9.

[18] Lalle M, Bruschi F, Castagna B, Campa M, Pozio E, Cacciò SM. High genetic polymorphism among Giardia duodenalis isolates from Sahrawi children. Trans R Soc Trop Med Hyg 2009;103:834-8.

[19] Ferreira FS, Centeno-Lima S, Gomes J, Rosa F, Rosado V, Parreira R, et al. Molecular characterization of Giardia duodenalis in children from the Cufada Lagoon Natural Park, Guinea-Bissau. Parasitol Res 2012;111:2173-7.

[20] Sprong H, Cacció SM, van der Giessen JW. Identification of zoonotic genotypes of Giardia duodenalis. PLoS Negl Trop Dis 2009;3:1-12.

[21] Cacciò SM, Ryan U. Molecular epidemiology of giardiasis. Mol Biochem Parasitol 2008;160:75-80. 